\title{
Diagnósticos de enfermagem de alcoolistas internados em uma unidade de saúde
}

Recebido em: 31/03/2013

Aprovado em: 16/05/2014

\author{
Mirella Carneiro Galvão' \\ Maria Angélica Bezerra dos Santos² \\ Marcos Venícios de Oliveira Lopes ${ }^{3}$ \\ Jaqueline Galdino Albuquerque Perrelli ${ }^{4}$ \\ Suzana de Oliveira Mangueira ${ }^{5}$
}

Resumo: Pesquisa descritiva, quantitativa, com a finalidade de identificar diagnósticos de enfermagem e a congruência de sua escrita em relação à NANDA Internacional e correlacioná-los com as necessidades humanas básicas. A partir dos prontuários de 131 alcoolistas internados em uma unidade hospitalar, verificou-se a presença de 72 diagnósticos distintos. O mais frequente foi “Risco de quedas" (12,2\%). As necessidades psicobiológicas foram as mais afetadas. Evidenciou-se a falta de registro de diagnósticos em $22,1 \%$ dos prontuários, bem como o descompasso de registros com a taxonomia, o que indica a necessidade de capacitação e de educação continuada dos enfermeiros do serviço.

Descritores: Alcoolismo; Processos de Enfermagem; Diagnóstico de Enfermagem.

\section{Nursing diagnoses of alcoholics admitted in a health unit.}

Abstract: Descriptive and quantitative research in order to identify nursing diagnoses and the congruence of his writing in relation to NANDA International and to correlate them with basic human needs. From medical records of 131 alcoholic admitted to hospitals, we verified the presence of 72 distinct diagnoses. The most common was "risk for falls" (12.2. The physiological needs were most affected. We observe a lack of registration of diagnoses in $22.1 \%$ of records, as well as the mismatch of records with the taxonomy of NANDA-I, which indicates the need for training and continuing education of nurses.

Descriptors: Alcoholism; Nursing Process; Nursing Diagnosis.

\section{Diagnósticos de enfermería de alcohólicos ingresados en una unidad de salud}

Resumen: Investigación descriptiva y cuantitativa a fin de identificar diagnósticos de enfermería y la congruencia de sus escritos en relación a la NANDA Internacional y correlacionarlos con las necesidades humanas básicas. A partir de los registros médicos de 131 alcohólicos ingresados en hospitales, se verificó la presencia de 72 diagnósticos distintos. El más común fue "riesgo de caídas" (12,2\%). Las necesidades fisiológicas son los más afectados. Se evidenció la falta de registro de diagnósticos en un $22,1 \%$ de los registros, bien como la falta de coincidencia de registros con la taxonomía de la NANDA-I, lo que indica la necesidad de capacitación y educación continua de los enfermeros de la institución. Descriptores: Alcoholismo; Procesos de Enfermería; Diagnóstico de Enfermería.

\section{INTRODUÇÃO}

$\mathrm{O}$ consumo abusivo do álcool impõe às sociedades de todos os países uma carga global de agravos negativos e caros e se constitui em um grave problema de saúde pública. Estimativas apontam que no Brasil entre $6 \%$ e $8 \%$ da população necessita de atendimento regular para os transtornos relacionados ao uso prejudicial de álcool e outras drogas $s^{(1)}$.

De todas as mortes no mundo, $4 \%$ são atribuídas ao álcool, percentual maior do que as mortes causadas por HIV/AIDS, violência ou tuberculose. $O$ cenário epidemiológico mostra que há expansão do consumo de algumas substâncias psicoativas, especialmente álcool e cocaína. É necessário assegurar um enfrentamento eficaz, competente e ancorado nos direitos de cidadania de dependentes, usuários, seus familiares e a população em geral (2).

Assim, a atenção do profissional de enfermagem deve estar voltada ao ser humano no atendimento de suas necessidades psicobiológicas, psicossociais e psicoespirituais e não visar apenas a sua doença ou desequilíbrio ${ }^{(3)}$. Neste contexto, emerge a necessidade de estabelecer o Processo de Enfermagem na prática de cuidados de saúde em domicílios, instituições de saúde e comunidades ${ }^{(4)}$. A
"De todas as

mortes no

mundo, $4 \%$ são

atribuídas ao

álcool, percentual

maior do que as

mortes causadas

por HIV/AIDS" finalidade de sua implantação nas instituições hospitalares do Brasil é a de organizar o cuidado a partir da adoção de um método sistemático, além de proporcionar ao enfermeiro a (re) definição do seu espaço de atuação, do seu desempenho no campo da gerência em saúde e da assistência em enfermagem ${ }^{(5)}$. No intuito de atender às exigências do Conselho Federal de Enfermagem quanto à implementação do processo de enfermagem nas instituições de saúde, diversos obstáculos têm sido enfrentados ${ }^{(6,7)}$.

De acordo com a NANDA Internacional, o diagnóstico de enfermagem é um julgamento clínico do enfermeiro acerca das respostas do indivíduo a uma determinada circunstância de sua vida. São interpretações científicas de dados que irão subsidiar a sequência de cuidados ao paciente ${ }^{(8)}$. Estudar os diagnósticos de enfermagem é relevante 
para a manutenção e o aperfeiçoamento da taxonomia da NANDA-I, bem como para fortalecer a prática clínica do enfermeiro, porquanto os diagnósticos subsidiam o estabelecimento das intervenções de enfermagem a avaliação ${ }^{(9)}$.

Entretanto, a implementação eficaz do processo de enfermagem é dificultada por alguns fatores, como a falta de conhecimento de todos os passos envolvidos no processo, excesso de tarefas para a equipe de enfermagem, má qualidade da formação profissional e registro insuficiente sobre o exame físico relacionado à doença em questão. As deficiências perpassam, principalmente, pela coleta de dados, registros de enfermagem e, consequentemente, pela ausência de diagnósticos ${ }^{(4)}$.

O estudo teve por objetivo analisar os diagnósticos de enfermagem em alcoolistas internados em um serviço de saúde e a congruência de sua escrita em relação à NANDA Internacional 2012-2014 e correlacionar os diagnósticos de enfermagem com as Necessidades Humanas Básicas.

\section{METODOLOGIA}

Pesquisa descritiva, com uma abordagem quantitativa, que teve como cenário de estudo um hospital filantrópico, que presta assistência interdisciplinar a alcoolistas, na unidade de cuidados prolongados. A unidade tem capacidade de 39 leitos.

A população do estudo foi composta pelos prontuários de pacientes diagnosticados com a Síndrome da Dependência Alcoólica, admitidos no ano de 2011. A amostra foi constituída por 131 prontuários que estavam disponíveis para consulta.

Por meio de um formulário construído pelos pesquisadores, buscou-se nos prontuários dados para a caracterização sóciodemográfica dos pacientes e o histórico de enfermagem utilizado pelo serviço, o qual é composto pelo registro da anamnese e exame físico, diagnósticos de enfermagem e intervenções. Os diagnósticos de enfermagem foram transcritos para posterior análise. Os dados obtidos foram analisados e armazenados em uma planilha do software Excel versão 2007. Foi realizado o cruzamento dos diagnósticos encontrados nos prontuários com a NANDA-I e verificada a congruência de sua escrita. Os diagnósticos constantes na NANDA-I foram analisados quanto à sua definição e foi feita a correlação com a definição das Necessidades Humanas Básicas ${ }^{(3)}$.

O estudo contou com a anuência da instituição e foi submetido ao Comitê de Ética em Pesquisa do Centro de Ciências da Saúde da Universidade Federal da Paraíba, o qual obteve aprovação, sob protocolo $\mathrm{n}^{\circ}$ 022/2012 e CAAE: 01133712.9.0000.5188.

\section{RESULTADOS}

Após análise dos dados obtidos, observou-se que 95,3\% da amostra foi composta por pacientes do sexo masculino, na faixa etária de 41 a 50 anos de idade (33,3\%). Verificou-se que em 102 (77,8\%) prontuários havia registro de diagnóstico de enfermagem e em 29 (22,1\%) o espaço destinado a tal registro estava em branco. Nos 102 prontuários foram identificados 72 diagnósticos de enfermagem distintos. Destes, 56 (77,7\%) estavam redigidos de acordo com a padronização da NANDA-I, $6(8,31 \%)$ estavam relacionados a diagnósticos de enfermagem da NANDA-I, mas encontravam-se com escrita incongruente e $10(13,8 \%)$ não estavam padronizados e nem possuíam diagnósticos equivalentes na referida taxonomia.

Dentre os diagnósticos redigidos de acordo com a NANDA, os mais frequentes foram: risco de quedas, nutrição desequilibrada: menos do que as necessidades corporais e risco de infecção, conforme mostra a Tabela 1.
Tabela 1 - Diagnósticos de enfermagem padronizados pela NANDA-I de maior frequência em pacientes alcoolistas internados. Vitória de Santo Antão- PE, 2012

\begin{tabular}{|ccc|}
\hline Diagnósticos de Enfermagem & N & $\%$ \\
\hline $\begin{array}{c}\text { Risco de quedas } \\
\begin{array}{c}\text { Nutrição desequilibrada: } \\
\text { menos do que as } \\
\text { necessidades corporais }\end{array}\end{array}$ & 28 & 12,2 \\
\hline Risco de infecção & 18 & 9,2 \\
\hline Dentição prejudicada & 14 & 7,8 \\
\hline $\begin{array}{c}\text { Insônia } \\
\text { Integridade da pele } \\
\text { prejudicada }\end{array}$ & 14 & 6,1 \\
Ansiedade & 10 & 6,1 \\
\hline Deambulação prejudicada & 8 & 4,3 \\
Outros & 117 & 3,5 \\
\hline
\end{tabular}

Outros diagnósticos de enfermagem foram identificados, porém, com menor frequência: estilo de vida sedentário, volume de líquidos excessivo, processos familiares disfuncionais, mobilidade física prejudicada, disposição para aumento da esperança, confusão aguda, disposição para religiosidade aumentada, disposição para aumento do autocuidado, entre outros.

Foi realizada a correlação dos 62 diagnósticos de enfermagem padronizados pela NANDA-I com a Teoria das Necessidades Humanas Básicas (NHB)(3). Verificou-se que as NHB mais afetadas foram as psicobiológicas, com 37 diagnósticos de enfermagem, seguidas pelas necessidades psicossociais, com 22 e as necessidades psicoespirituais, com 3 diagnósticos.

Dentre as necessidades humanas básicas psicobiológicas, as mais comprometidas foram: sono e repouso; integridade cutâneo-mucosa; regulação: térmica, hormonal, neurológica, hidrossalina, eletrolítica, imunológica, crescimento celular e vascular, como mostra a Tabela 2.

Tabela 2 - Número de diagnósticos de enfermagem de acordo com as necessidades humanas básicas psicobiológicas mais afetadas. Vitória de Santo Antão- PE, 2012

\begin{tabular}{|ccc|}
\hline NHB Psicobiológicas & $\begin{array}{c}\mathbf{N}^{\circ} \text { de } \\
\text { diagnósticos }\end{array}$ & $\%$ \\
\hline Sono e repouso & 5 & 13,5 \\
\hline Integridade cutâneo-mucosa & 5 & 13,5 \\
\hline Regulação & 5 & 13,5 \\
\hline Eliminação & 4 & 10,8 \\
\hline Hidratação & 3 & 8,1 \\
\hline Exercício e atividade física & 3 & 8,1 \\
\hline Nutrição & 3 & 8,1 \\
\hline Locomoção & 2 & 5,4 \\
\hline Integridade física & 2 & 5,4 \\
\hline Mecânica corporal & 2 & 5,4 \\
\hline Percepção & 2 & 5,4 \\
\hline Oxigenação & 1 & 2,8 \\
\hline Total & 37 & 100 \\
\hline
\end{tabular}

As necessidades humanas básicas psicossociais afetadas foram: gregária (36,3\%), autoestima $(18,1 \%)$, segurança $(13,6 \%)$, aprendizagem (9,0\%), participação $(9,0 \%)$, comunicação 
(4,5\%), recreação (4,5\%) e orientação no tempo e espaço (4,5\%). Dentre as NHB psicoespirituais, somente foi afetada a'religiosa e teológica' com $100 \%$ dos diagnósticos de enfermagem.

\section{DISCUSSÃO}

São muitas as dificuldades enfrentadas pelos enfermeiros na realização do processo de enfermagem. Quanto ao conhecimento sobre as etapas da SAE, um estudo evidenciou que os enfermeiros apresentaram respostas incompletas e poucos $(31,5 \%)$ descreveram todas as etapas corretamente. Em relação aos diagnósticos de enfermagem, o referido estudo constatou que a maioria (68\%) não citou nenhum diagnóstico e não os utilizava na prática profissional, devido a déficit de conhecimento sobre os mesmos ${ }^{(10)}$. Outra pesquisa apontou que os enfermeiros ainda necessitam melhorar a forma como estão descrevendo o diagnóstico de enfermagem, haja vista que foi identificado um descompasso dos registros em relação à evolução de enfermagem. A evolução também não apresentou conformidade em relação ao diagnóstico e prescrição de enfermagem ${ }^{(11)}$.

Observou-se, no serviço, a existência de prontuários sem diagnósticos de enfermagem registrados $(22,1 \%)$, bem como a presença de diagnósticos de enfermagem não padronizados pela taxonomia da NANDA-I ou com a escrita incorreta, que evidencia o déficit de conhecimento dos profissionais acerca da implementação adequada do processo diagnóstico em enfermagem.

Observou-se na Tabela 1 que os diagnósticos de enfermagem com maior frequência foram Risco de quedas (12,2\%) e Nutrição desequilibrada: menos do que as necessidades corporais (9,2\%).

O abuso de álcool pode gerar efeitos tóxicos no aparelho vestibular, além de causar degeneração cerebelar. Como consequência, pode apresentar tonturas e vertigens e, assim, comprometer o equilíbrio postural, de modo a afetar diretamente o organismo humano. $\mathrm{O}$ indivíduo apresenta instabilidade ao andar, com expressão de uma marcha atáxica com base ampla, além de dificuldades na execução de testes simples de equilíbrio(12).

Geralmente, entre os alcoolistas crônicos, a ingestão alimentar não é feita de forma que supra a necessidade calórica e gera uma diminuição nutricional de micronutrientes. Este déficit pode ser relacionado à anorexia ou a má absorção intestinal e favorece ao aparecimento de anemia e imunossupressão(13).

Outros diagnósticos encontrados nos prontuários de alcoolistas foram "Risco de infecção", "Dentição prejudicada" e "Insônia". O risco de infecção pode estar associado ao déficit nutricional ou mesmo ao processo de internação hospitalar. Quanto à dentição prejudicada, a literatura aponta que etilistas têm erosão dental devido às desordens somáticas, decorrentes dos frequentes quadros de refluxos, regurgitações e vômitos que são considerados fatores etiológicos intrínsecos, em virtude do contato recorrente dos ácidos endógenos com os dentes ${ }^{(14)}$. Já a insônia é um sintoma frequentemente presente entre os alcoolistas internados, devido ao fato de estarem em abstinência do álcool. A insônia ou inversão do ciclo do sono é um sintoma do alcoolismo relatado na literatura ${ }^{(15)}$.

Dos oito diagnósticos de enfermagem mais prevalentes, cinco corroboram os diagnósticos identificados em um estudo anterior que objetivou traçar o perfil de um grupo de pacientes atendidos num programa terapêutico para o alcoolismo e avaliar aspectos relacionados à qualidade assistencial por meio do processo de enfermagem, os quais foram: Nutrição desequilibrada: menos do que as necessidades corporais, Dentição prejudicada, Insônia, Integridade da pele prejudicada e Ansiedade, de modo a confirmar a frequência elevada destes diagnósticos em alcoolistas. Além disso, demonstrou-se que as NHB mais afetadas também foram as psicobiológicas, fato que necessita de intervenções condizentes com os problemas encontrados ${ }^{(16)}$.

A capacitação dos enfermeiros para a realização correta dos diagnósticos de enfermagem e das demais etapas do processo ainda é uma barreira que necessita ser transposta. Acredita-se que o conhecimento acerca da prevalência dos diagnósticos de enfermagem em determinadas populações contribui para a organização da base de conhecimento da profissão, para a previsão dos cuidados necessários e para a orientação de programas de educação continuada(17).

\section{CONCLUSÃO}

De acordo com a análise feita dos diagnósticos de enfermagem em prontuários de alcoolistas internados em um serviço de saúde e sua adequação em relação à NANDA Internacional 2009-2011, verificou-se que havia a falta de registro em alguns prontuários e ainda a presença de registros redigidos incorretamente ou não padronizados de acordo com a taxonomia. Tais aspectos indicam a necessidade de capacitação dos enfermeiros do serviço, no tocante ao processo de raciocínio diagnóstico e pensamento crítico, bem como quanto ao manuseio da taxonomia.

Os diagnósticos de enfermagem mais frequentes foram "Risco de quedas" e "Nutrição desequilibrada: menos do que as necessidades corporais" e as NHB psicobiológicas foram as mais afetadas. Estes dados mostram a necessidade do enfermeiro buscar compreender as bases fisiopatológicas destes fenômenos nos alcoolistas, de modo a embasar e direcionar o seu plano de cuidados. Ressalta-se ainda, a necessidade de buscar investigar as necessidades psicossociais e psicoespirituais, de modo a clarificar se realmente estas são menos frequentes na população em estudo ou estão sendo subvalorizadas e não registradas.

Os achados deste estudo limitam-se aos registros em prontuários de alcoolistas institucionalizados em um serviço de saúde. Portanto, estudos futuros que busquem investigar os diagnósticos de enfermagem em alcoolistas em outros cenários de prática devem ser encorajados, de modo a buscar identificar as necessidades mais afetadas nesta clientela e, assim, embasar a assistência de enfermagem voltada a ela. 


\section{Referências}

1. Vargas D. Versão reduzida da escala de atitudes frente ao álcool, alcoolismo e ao alcoolista: resultados preliminares. Rev Esc Enferm USP. 2011;45(4): 918-25.

2. Brasil. Ministério da Saúde. Secretaria de Atenção à Saúde. Plano emergencial de ampliação do acesso ao tratamento e prevenção em álcool e outras drogas. Brasília; 2009.

3. Horta WA. Processo de enfermagem. Rio de Janeiro: Guanabara Kooga; 2011.

4. Pokorski S, Moraes MA, Chiarelli R, Costanzi AP, Rabelos ER. Processo de enfermagem: da literatura à prática. O quê de fato nós estamos fazendo? Rev Latino-Am Enfermagem. 2009;17(3):302-7.

5. Castilho NC, Ribeiro PC, Chirelli MQ. A implementação da sistematização da assistência de enfermagem no serviço de saúde hospitalar do Brasil. Texto Contexto Enferm. 2009;18(2):280-9.

6. Mangueira SO, Lima JTS, Costa SLA, Nóbrega MML, Lopes MVO. Implantação da sistematização da assistência de enfermagem: opinião de uma equipe de enfermagem hospitalar. Enferm. foco. 2012;3(3):135-8.

7. Barros ALBL, Lopes JL. A legalização e a sistematização da assistência de enfermagem. Enferm Foco. 2010;1(2):63-5.

8. Herdman TH (Ed). Diagnósticos de enfermagem da NANDA: definições e classificação 2012-2014. Porto Alegre: Artmed; 2013.

9. Chaves ECL, Carvalho EC, Hass VJ. Validação do diagnóstico de enfermagem Angústia Espiritual: análise por especialistas. Acta Paul Enferm. 2010;23(2):264-70.
10. Silva EGC, Oliveira VC, Neves GBC, Guimarães TMR. O conhecimento do enfermeiro sobre a Sistematização da Assistência de Enfermagem: da teoria à prática. Rev Esc Enferm USP. 2011;45(6):1380-6.

11. Franco MTG, Akemi EN, D Inocento M. Avaliação dos registros de enfermeiros em prontuários de pacientes internados em unidade de clínica médica. Acta Paul Enferm. 2012;25(2):163-70.

12. Schmidt PMS, Giordani AM, Rossi AG, Cóser PL. Avaliação do equilíbrio em alcoólicos. Rev Bras Otorrinolaringol. 2010;76(2):14855.

13. Maio R, Dichi JB, Burini RC. Implicações do alcoolismo e da doença hepática crônica sobre o metabolismo de micronutrientes. Arq Gastroenterol. 2010;37(2):120-4.

14. Catelan A, Guedes APA, Santos PH. Erosão dental e suas implicações sobre a saúde bucal. RFO UPF. 2010;15(1):83-6.

15. Chagas M. Hildebrandt LM, Leite MT, Stumm EMF, Vianna RM. O alcoolismo e o grupo de alcoólicos anônimos: o conhecimento de alcoolistas. Cad Bras Saúde Mental. 2010;2(4-5):190-212.

16. Souza RS, Siqueira MM. O processo de enfermagem na assistência a pacientes com dependência de álcool. J Bras Psiquiatr. 2005;54(3):228-33.

17. Paganin A, Menegat P, Klafke T, Lazzarotto A, Fachinelli TS, Chaves IC, et al. Implantação do diagnóstico de enfermagem em unidade de terapia intensiva: uma análise periódica. Rev Gaúcha Enferm. 2010;31(2):307-13. 